\title{
EMITTANCE GROWTH BY SYNCHROTRON RADIATION IN A DOUBLE-SIDED MICROTRON*
}

\author{
J. Herrmann, K.-H. Kaiser", S. Ratschow \\ Institut fuer Kernphysik, J.-Gutenberg Universitaet, D-55099 Mainz, Germany \\ V.I. Shvedunov \\ Institute of Nuclear Physics, Moscow State University, R-119899 Moscow, Russia
}

\begin{abstract}
Here we present results of calculations of emittance growth caused by quantum fluctuations of synchrotron radiation (QFSR) for a $1.5 \mathrm{GeV}$ Double-Sided Microtron (DSM) [1]. We did both semi-analytical estimations, employing known twiss parameters for the DSM orbits, and a computer simulation of these stochastical effects using the program SYTRACE [2]. This showed that the normalized emittance growth was within reasonable limits, by a factor of about 1.5 , thus permitting e.g. the installation of small aperture linacs on the DSM axes.
\end{abstract}

\section{INTRODUCTION}

A Double-Sided Microtron (DSM) is being planned to upgrade the three-staged cw racetrack microtron (RTM) cascade MAMI from $0.855 \mathrm{GeV}$ to $1.5 \mathrm{GeV}$. The DSM consists of two rf linacs interconnected by symmetrical pairs of $90^{\circ}$ segment magnets (s. fig.1). The scheme of the RTM is unpractical in this energy range because of the excessive weight of its end magnets. The relatively strong vertical defocusing by the $45^{\circ}$-pole face inclination at both the beam entrance and the exit of the segment magnets is compensated in the whole energy range by an appropriate field gradient normal to the pole edge [3]. Since the parallel-to-parallel optics in the horizontal plane is not affected by this two-dimensional field gradient, a pair of end magnets acts as energy-dependent effective drift spaces which are specific for the horizontal and vertical planes. Beam focusing is provided by four quadrupole doublets (two of which, $\mathrm{Q}_{1} \& \mathrm{Q}_{2}$ and $\mathrm{Q}_{3} \& \mathrm{Q}_{4}$, are shown in Fig. 1), installed between linacs and $90^{\circ}$ end magnets $\left(M_{1}\right.$, $\mathrm{M}_{2}$ ). The main parameters of the DSM are given in Table I.

As a consequence of the field gradient, the coherent energy gain per turn is no longer constant, leading to a shift of the central bunch phase during the acceleration process. Investigations of the longitudinal beam dynamics have shown that the stability with respect to small deviations of the accelerator parameters can be improved significantly by operating one of the linacs at the first subharmonic of the DSM frequency. This is possible because at our subharmonic injection (the MAMI-RTMs are operating at half the DSM-frequency), only every second wave crest is occupied in one of the two DSM linacs.

For both the design of the DSM and the use of its beam in nuclear and particle physics experiments it is extremely important to make reliable predictions about the emittance of the beam. Horizontal emittance growth by QFSR was calculated and confirmed by measurements [4] for the third microtron (RTM3). QFSR induced longitudinal and horizontal emittance growth for general polytron scheme has been estimated in [5].

We used a simplified semi-analytical approach to obtain horizontal emittance dependence on the beam energy and to estimate beam losses. Computer simulation with particle tracking code SYTRACE takes into account the bending magnets field gradient, the possible beam mismatch, the change of beam ellipse parameters under synchrotron radiation and the nonlinear distortions of longitudinal phase space.

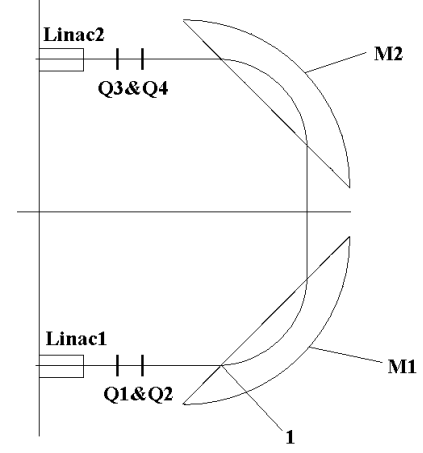

Figure 1. Half of DSM.

Table I. DSM main parameters.

\begin{tabular}{|l|l|}
\hline Injection energy & $0.855 \mathrm{GeV}$ \\
\hline Maximum output energy & $1.5 \mathrm{GeV}$ \\
\hline Number of orbits & 43 \\
\hline Energy gain per turn & $16.58-13.66 \mathrm{MeV}$ \\
\hline Linac 1 frequency & $2.4495 \mathrm{GHz}$ \\
\hline Linac 2 frequency & $4.899 \mathrm{GHz}$ \\
\hline Field induction & $1.53-0.95 \mathrm{~T}$ \\
\hline
\end{tabular}

${ }^{*}$ Work supported in part by DFG (SFB 201)

${ }^{\#}$ kaiser@kph.uni-mainz.de 


\section{HORIZONTAL EMITTANCE}

\subsection{Semi-analytical estimations}

Supposing strict mirror symmetry of the DSM optical scheme in canonical coordinates, uniform end magnet field and following [6], [7] we obtain the next estimate for the average emittance growth after passage of one pair of end magnets at the $i$-th half-turn:

$$
\Delta\langle\varepsilon\rangle_{i}=\frac{\left\langle\Delta E^{2}\right\rangle_{i}}{E^{2}{ }_{i}}\left[\frac{\pi}{2} \beta_{1 i}-2 r_{i} \alpha_{1 i}+\left(\frac{3 \pi}{2}-4\right) r^{2}{ }_{i} \gamma_{1 i}\right],
$$

where: $\left\langle\Delta E^{2}\right\rangle_{i}$ is the mean square beam energy spread due to synchrotron radiation per unit of bending angle, $E_{i}$ beam energy and $\beta_{1 i}, \alpha_{1 i}, \gamma_{1 i}$ - horizontal plane twiss parameters at point 1 (Fig. 1), $i=1,2, \ldots 2 N, N$ - total number of turns.

$$
\frac{\left\langle\Delta E^{2}\right\rangle_{i}}{E_{i}^{2}}=\frac{55}{96 \sqrt{3}} \frac{e^{4} \hbar}{\pi \varepsilon_{0} c\left(m_{0} c\right)^{4}} B^{2} \gamma_{i}^{3} \approx 4.96 \times 10^{-22} B^{2} \gamma_{i}^{3}
$$

where $\mathrm{B}$ is field in $\mathrm{T}$ and $\gamma_{i}$ - relative beam energy.

Formula (1) takes into account that pairs of DSM end magnets are achromatic. Twiss parameters at point 1 are obtained from the known twiss parameters at the linac1 center (see Fig. 2) through matrix transformations involving matrices for the half linac, the drift spaces, the quadrupole singlets and the $45^{\circ}$ pole face rotation at the entrance of $\mathrm{M}_{1}$.

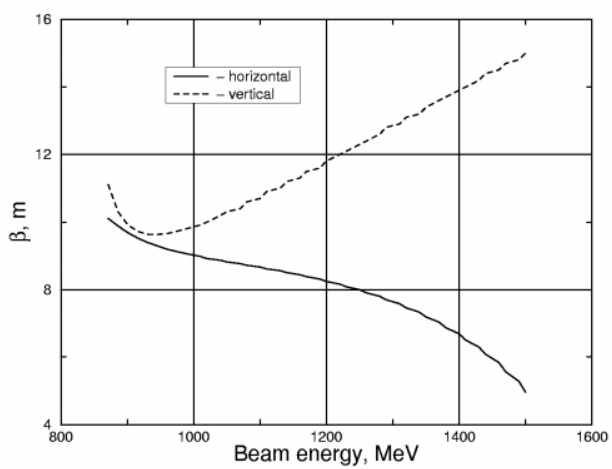

Figure 2. Energy dependence of the horizontal and vertical DSM $\beta$-functions at linac centers.

Taking into account the pseudo-damping due to acceleration while neglecting other sources of emittance growth, the average emittance after $n$ turns is:

$$
\langle\varepsilon\rangle_{n} \approx\langle\varepsilon\rangle_{0} \frac{\gamma_{0}}{\gamma_{2 n}}+\frac{1}{\gamma_{2 n}} \sum_{i=1}^{2 n} \Delta\langle\varepsilon\rangle_{i} \gamma_{i},
$$

where $\langle\varepsilon\rangle_{0}$ and $\gamma_{0}$ are emittance and relative energy at injection.

In Fig. 3 the dependence of DSM horizontal emittance on energy is shown, calculated with formulas (1)-(3). The initial value of average emittance at $0.855 \mathrm{GeV}$ was taken to be $0.0107 \pi m m \times \operatorname{mrad}$ [4]. Thus total growth of the normalized horizontal emittance is by a factor of about 1.5.

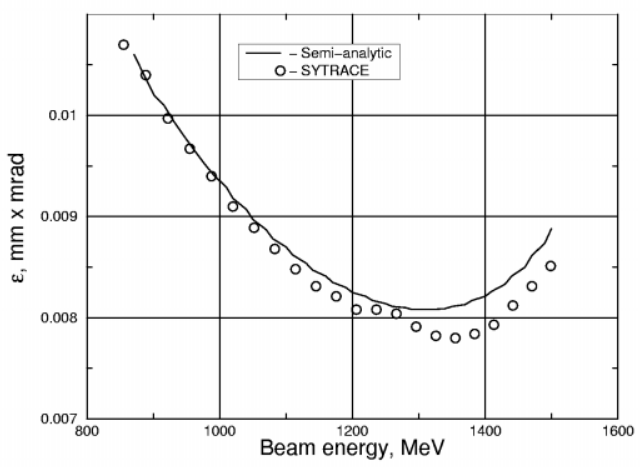

Figure 3: Horizontal emittance behaviour in DSM.

For "clean" DSM operation, relative particle losses during acceleration must be of less than $10^{-5}$. To obtain estimations of the aperture radius $b_{n}(\eta)$, providing the transmission of a certain fraction $\eta$ of the beam current at some specific point along the accelerator at the $n$-th turn, we use:

$$
b_{n}(\eta)=\sqrt{\langle\varepsilon\rangle_{n} \beta_{n}^{*}} \Phi^{-1}(\eta),
$$

here $\beta_{n}^{*}$ - horizontal beta function at a considered point and $\Phi^{-1}(\eta)$ - inverse error function.

The most dangerous positions in DSM from the point of view of beam losses are the linac ends, where $\beta$-function $\beta_{l e}$ is close to maximum and is connected with that at linac center, shown at Fig. 2 by $\beta_{l e} \approx \beta+L_{1 / 2}^{2} / \beta$, where $L_{1 / 2}$ is half linac length. Beam aperture radii for transmission of a certain beam current fraction, calculated with formula (5), are shown in Fig. 4. In spite of emittance growth, starting from about $1300 \mathrm{MeV}$, beam limiting aperture steadily decreased with energy because of the horizontal $\beta$-function $\beta_{l e}$ decrease. The estimated minimum beam aperture radius for beam losses at the level of $10^{-5}$ is less than $1.2 \mathrm{~mm}$, while the beam hole radius for the $4.9 \mathrm{GHz}$ linac will be $5 \mathrm{~mm}$.

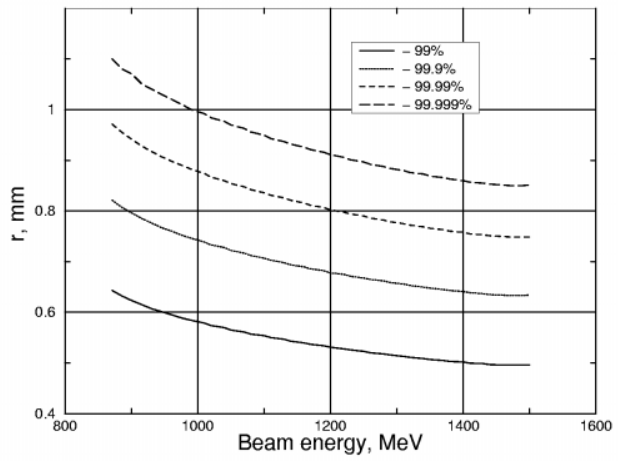

Figure 4. Beam aperture radius for transmission of different fractions of beam current. 


\subsection{Results of SYTRACE simulation}

SYTRACE [2] is an extension of the well-proven PTRACE [8] code, used for the design of the MAMI RTMs. It includes the option to simulate a particle ensemble behaviour with QFSR taken into account. A precise integration of the beam dynamics equations is performed for each DSM element.

When integrating the equations of motion in the end magnets field, particle energy losses due to the emission of photons are taken into account. At each step for each particle, a photon can be emitted with a probability defined by the particle energy, local trajectory curvature and value of step size. Subsequently to each emitted photon an energy is prescribed, according to the probability function produced from the photon number spectrum. Because the photon number spectrum is divergent for relative photon energy $\xi=\mu / \mu_{c} \rightarrow 0\left(\mu_{c}-\right.$ critical photon energy), it is truncated at $\xi_{t r} \approx 0.002$, producing an increase of $14 \%$ of the average energy $\langle\mu\rangle$ of emitted photons. The specific value of $\xi_{t r}$ is defined by limitations of the memory used to store the uniform step probability function. To have an average electron energy loss per turn $U=2 \pi\langle\mu\rangle\langle n\rangle$, in accordance with the theory, the average number of photons $\langle n\rangle$ emitted per unit angle is decreased by the same value. Because of $\left\langle\Delta E^{2}\right\rangle=275 / 64\langle\mu\rangle^{2}\langle n\rangle$ this procedure, according to (1), can lead to an emittance growth over-estimated by about $14 \%$.

Dependence on energy of the horizontal plane rms emittance, calculated with SYTRACE is shown in Fig. 3 by open circles. For the same values of initial emittance, the semi-analytical value at $1.5 \mathrm{GeV}$ is about $4 \%$ higher than the numerical one. Combined with the overestimation described above this means a difference of about $18 \%$. This difference can qualitatively be explained by the fact that for the semi-analytical calculations constant field magnets are used, whereas in the SYTRACE simulations the exact field decay could be taken into account. As can be seen from formula (2) the resulting decrease of the mean magnet field at high energies leads to smaller values for $\left\langle\Delta E^{2}\right\rangle_{i}$ and, consequently, to a smaller emittance growth.

\section{LONGITUDINAL EMITTANCE}

The energy spread caused by QFSR after the passage of the beam through a pair end magnets increases from about $2.2 \mathrm{keV}$ at the first DSM orbit to $8.8 \mathrm{keV}$ (rms) at the last. Total QFSR energy spread at N-th orbit is defined by:

$$
\delta E_{N}=\sqrt{\pi \sum_{i=1}^{2 N}\left\langle\Delta E^{2}\right\rangle_{i}}
$$

where $\left\langle\Delta E^{2}\right\rangle_{i}$ is given by (2), $\pi$ is bending angle of magnet pair, and for $N=43, \delta E_{43} \approx 50 \mathrm{keV}$. This energy spread is transformed to the phase spread with resulting longitudinal emittance growth. We estimate this growth supposing that the induced emittance is filling phase space eigenellipse. From numerical simulations a semi-axes ratio of $\delta E_{43} / \delta \varphi_{43} \approx 35 \mathrm{keV} / \mathrm{deg}$ is obtained for the eigenellipse in the middle of the bending systems. This leads to an estimated total emittance growth of about 35 $\mathrm{keV} \times$ deg. which is in good agreement with the result of SYTRACE simulations shown in Fig. 5.

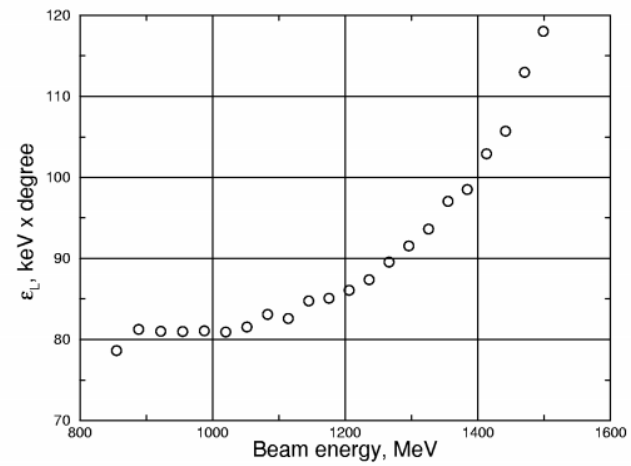

Fig. 5. Longitudinal emittance from SYTRACE. (The phase unit is related to $4.9 \mathrm{GHz}$.)

\section{CONCLUSION}

We presented results of semi-analytical and numerical calculations of emittance growth caused by QFSR in a 1.5 $\mathrm{GeV}$ double sided microtron. Estimated normalised rms horizontal emittance at maximum energy is about $25 \pi$ $\mathrm{mm} \times \mathrm{mrad}$, while longitudinal emittance is about 120 keV $\times$ degree.

\section{REFERENCES}

[1] H.Herminghaus K.-H. Kaiser, U. Ludwig-Mertin, "The Design of Double-Sided Microtron"; Proc. of the 1981 Linear Acc. Conf., Santa Fe, N.M., LA-9234-c, p.260.

[2] J.Herrmann, S.Ratschow; development based on PTRACE

[3] K.-H. Kaiser, "A Possible Magnet Field Configuration for a C.W. Electron Accelerator in the GeV Region", Proc. of the Conf. On Future Possibilities for Electron Accelerators, Charlottesville, VA, 1979, p. V-1.

[4] H. Euteneuer, F. Hagenbuck, R. Herr, J. Hermann, K. Herter, M.O. Ihm, K.H. Kaiser, Ch. Klumper, H.-P. Krahn, H.-J. Kreidel, U. Ludwig-Mertin, G. Stephan, Th. Weiss, "Beam Measurements and Operating Experience at MAMI", Proc. of the 1994 European Particle Acc. Conf., London, 1994, p.. 506.

[5] H. Herminghaus, "The Polytron as a CW Electron Accelerator in the 10 GeV Range", NIM A305(1991)1

[6] Crosbie "Synchrotron Radiation Effects in the Argonne Hexatron", Argonne, 1983, ANL-GEM-40-83

[7] M. Sands, "The Physics of Electron Storage Rings", SLAC-121, 1970

[8] K.-H. Kaiser, PTRACE (written on the base of RAYTRACE from S.A. Enge and S. Kowalski), private communication 